\title{
Capsule Commentary on Warner et al., Faculty Promotion and Attrition: the Importance of Coauthor Network Reach at an Academic Medical Center
}

\author{
José E. Rodríguez, MD \\ The Center for Underrepresented Minorities in Academic Medicine, The Florida State University College of Medicine, Tallahasse, FL, USA.
}

J Gen Intern Med 31(1):104

DOI: $10.1007 / \mathrm{s} 11606-015-3497-\mathrm{x}$

(c) Society of General Internal Medicine 2015

$\mathrm{U}$ nderrepresented Minorities in Medicine (URMM) (consisting of Blacks, Latinos and Native Americans) continue to be poorly represented in medical faculties across the USA. ${ }^{1}$ While the cause of this is multifactorial, Dr. Warner and colleagues examine one aspect of faculty promotion and attrition at Harvard Medical School, coauthor network reach. ${ }^{2}$ For 4 years they studied coauthor network reach and then compared URMM faculty to White faculty to determine differences. Men and Whites were found to have significantly larger coauthor networks when compared to women and URMM faculty members. They also found a positive correlation between network reach and promotion, as well as retention. These findings at HMS reflect what URMM faculty have experienced for decades - a lack of mentoring and isolation.

Research on URMM faculty suggests that many disparities including racism, isolation, lack of mentorship, clinical disparities, diversity efforts disparities and promotion disparities negatively affect URMM faculty and are significant barriers to increasing URMM faculty presence. ${ }^{3}$ Among the solutions suggested to improve URMM representation is improving URMM networking opportunities. ${ }^{3}$ Dr. Warner and colleagues are to be commended for quantifying this phenomenon. Providing solid evidence and statistics, her team has made it clear that improving network reach is one solution to the problem of URMM attrition from medical faculties.

The study also begs the question of why do women and minorities have smaller networks? Is there a reason that URMM faculty have less network reach or is it an artifact of their low representation? And when URMM faculty have similar network reach as their non-URMM colleagues, will we see any alleviation in the promotion disparity? Dr. Warner and colleagues' work gives us at least one answer to this problem. Collaboration with URMM faculty is to be increased if we are to sustain the current representation of URMM faculty at medical schools in the US. Without it, there is not hope for population parity or even progress toward that goal.

Conflict of Interest: The author has no conflict of interest with this article.

Corresponding Author: José E. Rodríguez, MD; The Center for Underrepresented Minorities in Academic Medicine, The Florida State University College of Medicine, Tallahasse, FL, USA (e-mail: Jose.rodriguez@med.fsu.edu).

\section{REFERENCES}

1. Rodriguez JE, Campbell KM, Adelson WJ. Poor representation of Blacks, Latinos, and Native Americans in medicine. Fam Med. 2015;47(4):259-63.

2. Warner ET, Carapinha R, Weber GM, Hill EV, Reede JY. Faculty promotion and attrition: the importance of coauthor network reach at an Academic Medical Center. J Gen Intern Med. 2015. doi:10.1007/s11606-015-3463-7.

3. Rodriguez JE, Campbell KM, Pololi LH. Addressing disparities in academic medicine: what of the minority tax? BMC Med Educ. 2015;15(1):6.

Published online August 26, 2015 